\title{
The Evolving Role of Saline Infusion Sonography (SIS) in Infertality- Systemic Review
}

\author{
Muhammad Rohail Amjad* \\ The University of Lahore \\ 1-Km Defense Road Lahore, Pakistan \\ Hira Riffat \\ The University of Lahore \\ 1-Km Defense Road Lahore ,Pakistan \\ Saba Ajmal \\ The University of Lahore \\ 1-Km Defense Road Lahore ,Pakistan \\ Syeda Khadija-Tul-Sughra \\ The University of Lahore \\ 1-Km Defense Road Lahore ,Pakistan
}

The research is financed by Asian Development Bank. No. 2006-A171(Sponsoring information)

\begin{abstract}
Background: To perform a systematic review to examine all the available literature reporting to describe the Structural intrauterine abnormalities are an important cause of infertility, recurrent pregnancy loss and bleeding or pain associated with a poor reproductive outcome. Various diagnostic methods have been applied to detect these lesions such as hysterosalpingography, hysteroscopy and Sonohysterography.Materials and methods: In this systematic review data extracted by Medline, PubMed, science direct and Obs gyne online library that were queried for studies published between 2000 to 2020 by using specific MeSH terms. In this reviewed article we extracted data from 15 retrospective Cohort studies in which 3800 infertile women underwent SIS procedure. Infertile women were screened for possible uterine pathologies.Results: In this systematic review we reviewed 15 article that was published between 2000 to2020. Extracted data from these reviewed articles showed Two thousand infertile patients examined by saline infusion Sonography. From these 2000 patients 1400 women diagnose with abnormalities.Conclusion:Saline infusion sono-hysterography was found to be a more reliable method of diagnosing tubal or uterine pathologies in infertile patients.it is cost effective and gold standard.
\end{abstract}

Keywords: Intrauterine, Pathology, Sonohysterography, Saline infusion sonography

DOI: $10.7176 / \mathrm{JHMN} / 90-03$

Publication date:June $30^{\text {th }} 2021$

\section{Introduction}

Infertility (subfertility) is a problem that is increasing day by day. According to World Health Organization reports. Around $10 \%$ of women are facing problems of infertility globally. ${ }^{1}$ Etiology of infertility is multifactorial and fallopian tube abnormality being one of the most important causes accounts for up to $40 \%$ of female subfertility if not less and is further increasing. ${ }^{2}$ Hence, screening for tubal occlusion is one of the first important steps in fertility assessment of the investigation of sub fertile couples. Till date, hysterosalpingography (HSG) is the preferred choice of investigation followed by laparoscopy with chromotubation. ${ }^{3}$

Over the last three decades, saline infusion sonography (SIS) has become an integral part of ultrasound scanning in gynecology. SIS is a technique which enables better visualization of the uterine cavity than conventional transvaginal ultrasound. It is performed by introducing fluid gradually into the uterine cavity via a catheter placed through the cervix. ${ }^{4}$ This procedure is also known as Sonohysterography, Hyster sonography, hydrosonography or gel instillation sonography (GIS). ${ }^{5}$

Compared to hysteroscopy, SIS is relatively a shorter examination, less invasive, well tolerated by patients and more cost effective. The concept of intrauterine saline infusion synchronized with transvaginal sonography (TVS) was described by Nannini et al. after early experiences revealed improved uterine cavity images. ${ }^{4}$ The endometrial appearance, thickness and echogenicity vary with different phases of the menstrual cycle. Thus, small endometrial polyps or intrauterine adhesions could be missed on routine two-dimensional (2D) transvaginal ultrasound scan. Large submucosal myomas or multiple uterine fibroids can obscure views of the uterine cavity, and delineation of the endometrium is difficult in such cases. For these reasons, in some clinical scenarios diagnostic TVS cannot provide sufficient information. Other indications for SIS include abnormal uterine bleeding and recurrent miscarriage where the endometrium cannot be adequately assessed. In a normal SIS study, the 
endometrium appears symmetric, surrounding an anechoic, saline-distended uterine cavity. ${ }^{6}$

In 2003 the American College of Obstetricians and Gynecologists (ACOG), in conjunction with the American College of Radiology (ACR) and the American Institute of Ultrasound in Medicine (AIUM), developed a technology assessment document for saline infusion sonography. ${ }^{7,8}$ According to the AIUM's recommendations, SIS will aid evaluation of the uterine cavity in a number of conditions. ${ }^{9}$

The indications related to subfertility include:

- Infertility: especially in women with recurrent implantation failure following in vitro fertilization (IVF) cycles.

- Congenital abnormalities of the uterine cavity: although 3- dimensional (3D) ultrasound scanning is considered to be the gold standard for diagnosis of congenital abnormalities, SIS plays a role in the diagnosis of subseptate uterus.

- Preoperative or postoperative evaluation of uterine myomas and polyps.

- $\quad$ Suspected uterine cavity adhesions.

Contraindications to performing SIS

- Suspicion or diagnosis of current infection including Chlamydia, syphilis or gonorrhoea. This could potentially result in a pyosalpinx or tuboovarian abscess. Previous history of sexually transmitted infection may be an indication for prophylactic antibiotics.

- In women with a positive pregnancy test

The examination is best performed during the follicular phase (ideally before day 10) of the menstrual cycle, after menstrual flow has ceased. After this period, focal irregularities of the endometrial contour appear and could be misinterpreted as small polyps. ${ }^{10}$ Several purpose-designed catheters with balloons are commercially available (Angio tech catheter, 5.5 fr $40 \mathrm{~cm}$, Ref: 660005040, Canada) or (Cook catheters, Ref: J-CHSG-503000, USA), although it is possible to use embryo transfer catheters as cheaper alternatives. The fluid distends the cavity of the uterus and acts as a negative contrast agent to provide a detailed and well defined view of the endometrium and its surface contours. Sometimes the inflated balloon of the catheter may interfere with obtaining an optimal view of the cavity. Deflation of the balloon at the end of the procedure and further instillation of saline before the catheter comes out ensure an adequate view, particularly of the lower uterine cavity and cervical canal. ${ }^{11}$ SIS can be performed with conventional two dimensional (2D) or 3D ultrasound. With the 2D technique, the uterine cavity is evaluated while saline is being instilled. 3D ultrasound allows capture of a volume of information of the uterus within a few seconds as the saline is instilled into the uterine cavity and the dataset can be stored and subsequently re-analyzed after the procedure has been completed. This means that the procedure is short; less saline volume is needed and therefore, it has the potential to reduce patients' discomfort significantly. Further, the image planes obtained with 3D ultrasound, particularly the coronal plane, may provide additional diagnostic information of the uterine cavity and pathology. ${ }^{12}$

SIS is well tolerated by the vast majority of women. A randomized trial demonstrated that women experience less discomfort with SIS than hysteroscopy. It is good practice to instruct women to take pre-prescribed analgesia an hour prior to the procedure to reduce abdominal cramps, although studies have demonstrated no difference in the pain score between patients who received analgesia and placebo prior to the procedure. ${ }^{13}$ SIS is a diagnostic, not a therapeutic tool, thus necessitating the need for a second procedure, e.g. therapeutic hysteroscopy, for resection of intrauterine pathology. Technical difficulties can arise during SIS due to inability to pass the catheter through the cervix in women with cervical stenosis (failure rate $1.8-6 \%$ ). ${ }^{14,15}$ A clear image of the endometrial interface is obtained with the proper amount of intrauterine saline infusion.

\subsection{Method:}

\section{Search strategy:}

In this systematic review data extracted by Medline, PubMed, science direct and Obsgyne online library that were queried for studies published between 2000 to 2020 by using specific MeSH terms.

\section{Study selection:}

In this reviewed article we extracted data from 15 retrospective Cohort studies in which 3800 infertile women underwent SIS procedure. Infertile women was screened for possible utrine pathologies.

\subsubsection{Discussion}

Rubina Izhar Et al (2019) conducted a study on "Diagnostic accuracy of Saline Infusion sonohysterosalpingography (SIS) as compared to Hystero-salpingography (HSG) in the assessment of sub-fertile women" in a private infertility clinic in Karachi, and comprised women who registered for assessment of infertility from June 2011 to May 2013. Of the 256 subjects, 184 (72\%) presented with primary and 72 (28\%) with secondary infertility. The sensitivity of saline infusion sonohysterography for detecting patency of the tubes was $100 \%$ and the specificity was $91 \%$ whereas positive predictive value was $69.8 \%$ and negative predictive value was $100 \%$ (Kappa: 0.61 ). For large uterine polyps, sensitivity and specificity was $100 \%$ and for small polyps sono-hystero- 
salpingography showed $14 \%$ false positive results (Kappa: 0.21 ). Saline infusion sono-hysterography was found to be a more reliable method of diagnosing tubal or uterine pathologies compared to hystero-salpingography for cases of infertility. ${ }^{16}$

Vanita Singh Et al (2018) conducted a retrospective study on "Role of Saline Infusion Sonohysterography in Infertility Evaluation". The study was done on the basis of record maintained in the ultrasound register and case record sheets of all infertile women who underwent infertility treatment and fulfilled the inclusion criteria from June 2015 to August 2016.SIS had sensitivity of $91 \%$, specificity of $76 \%$ positive predictive value (PPV) of $95 \%$, negative predictive value (NPV) of $66 \%$, and accuracy of $89 \%$ of SIS in evaluating tubal patency. Further, SIS showed sensitivity of $83.3 \%$, specificity of $60 \%$, PPV of $75 \%$, NPV of $75 \%$, and accuracy of $72 \%$ in detecting pelvic pathology.In a low-resource country like India where patients are also less educated, SIS can prove to be a useful tool in initial workup of infertility patients with better compliance, low cost, and better results in a single visit. ${ }^{17}$

Samia Hussain Et al (2018) conducted a study on "Incidence of intrauterine abnormalities in Pakistani women with unexplained infertility diagnosed via saline infusion sonography". This was a retrospective cohort study conducted at Aziz medical centre, Karachi, Pakistan between January and December 2015. The study population comprised of women with unexplained infertility who underwent saline infusion sonography as a part of their diagnostic workup. The frequency of uterine abnormalities in these women was determined and the relationship between these pathologies and patient age and body mass index was assessed.Of the 769 women included, 202 (26.3\%) had uterine abnormalities. Endometrial polyp (118 cases, 15.3\%) was the most common abnormality, followed by submucous fibroids $(54,7 \%)$, intrauterine adhesions $(20,2.6 \%)$, and septae $(10,5 \%)$. Intrauterine pathologies were more common in women with primary infertility $(71.8 \%$ versus $28.2 \%, \mathrm{p}=0.002)$. Uterine abnormalities were most common in the age group 30-34 years $(n=80,39.6 \%)$ and in overweight patients $(n=$ $95,47 \%)$. The distribution of abnormalities differed significantly in various age groups $(\mathrm{p}=0.009)$ and among women with different BMI $(\mathrm{p}=0.029)$.A significant number of women with unexplained infertility present with unsuspected uterine abnormalities; therefore an assessment of the uterine cavity should be performed in all cases. ${ }^{18}$

Banu Bingol Et al (2010) conducted a study on "Comparison of diagnostic accuracy of saline infusion sonohysterography, transvaginal sonography and hysteroscopy in postmenopausal bleeding". This study was conducted on 137 postmenopausal women, with abnormal uterine bleeding, admitted to the Department of Obstetrics and Gynecology of Istanbul Bilim University, Florence Nightingale Hospital and Fertigyn Woman Health and IVF Center. After TVS, all patients underwent SIS using Cook Soft 500 IVF transfer catheter and HS, consecutively. Sensitivity, specificity, and positive and negative predictive values (PPV and NPV) were calculated to compare the diagnostic accuracy of TVS, SIS and HS.Most commonly encountered endometrial lesions were polypoid lesion $(38.0 \%)$ and hyperplasia $(28.4 \%)$ among our study population consisting of 137 women (mean age $61.6 \pm 9.6$ years) in their postmenopausal stage.Overall sensitivity rates were $70.0 \%$ for TVS, $89.6 \%$ for SIS and $92.3 \%$ for HS, while the overall specificity rates were 50.0, 77.3 and $80.7 \%$, respectively. HS had PPV of $96.2 \%$ and NPV of $65.3 \%$, whereas PPV was determined to be 80.9 versus $95.3 \%$ and NPV was 35.4 versus $58.3 \%$ for TVS and SIS, respectively.As an easy to perform, safe and well-tolerated procedure yielding high diagnostic accuracy, saline infusion SIS via this catheter seems to be superior to TVS and very close to HS. It may be used as the primary method for the detection of uterine abnormalities among postmenopausal women with abnormal uterine bleeding. ${ }^{19}$

Mohamed Rezk Et al (2014) conducted a study on "The safety and acceptability of saline infusion sonography versus hysterosalpingography for evaluation of tubal patency in infertile women"

In this study, 104 consecutive infertile women underwent SIS and HSG for tubal patency followed by laparoscopy with dye test as the gold standard test. Test parameters, safety and acceptability of both methods were assessed. SIS showed patency in $90(86.5 \%)$ tubes, HSG in $85(81.7 \%)$ tubes, and laparoscopy in $75(72.1 \%)$ tubes. SIS and laparoscopy agreed in 15 out of 29 occluded tubes (concordance, $51.7 \%$ ) while HSG and laparoscopy agreed in 11 out of 29 occluded tubes (concordance, 37.9\%). The sensitivity, specificity, PPV, NPV were 52\%, $95 \%, 79 \%, 84 \%$ for SIS and 38\%, 96\%, 79\%, 80\% for HSG respectively. There were a significant number of women who experienced pain, syncopy and cervicovaginal lacerations during HSG examination in comparison to SIS $(P<0.001)$. SIS was more acceptable than HSG as a screening test for tubal patency regarding the overall discomfort and the overall satisfaction rate.

Although HSG is the standard screening test for the diagnosis of tubal infertility, the present study confirmed the higher sensitivity, safety and acceptability of SIS compared to HSG for the evaluation of tubal patency in infertile women. ${ }^{20}$

B Bingol Et al (2011) conducted a study on "Comparison of diagnostic accuracy of saline infusion sonohysterography, transvaginal sonography and hysteroscopy". A total of 346 patients were selected for operative hysteroscopy, following SIS after TVS. SIS was performed with a Cook Soft 500 IVF catheter. Sensitivity, specificity, positive and negative predictive values (PPV, NPV) were calculated to compare the accuracy of TVS, SIS and hysteroscopy for uterine abnormalities. SIS showed a sensitivity of $87 \%$, specificity of $100 \%$ and PPV of 
$100 \%$ for endometrial hyperplasia, and a sensitivity and NPV of $100 \%$ for polypoid lesions. For submucosal myoma SIS showed a sensitivity of $99 \%$ with PPV of 96\%. Hysteroscopy had a sensitivity, specificity, PPV and NPV of $98 \%, 83 \%, 96 \%$ and $91 \%$, respectively for overall uterine pathologies. Finally, SIS seems to be superior to TVS, for uterine pathologies, with respect to hysteroscopy as the gold standard. ${ }^{21}$

Seshadri S Et al (2015) conducted a study on "Diagnostic accuracy of saline infusion sonography in the evaluation of uterine cavity abnormalities prior to assisted reproductive techniques". The pooled sensitivity of SIS in the detection of all intrauterine abnormalities was 0.88 (95\% confidence interval (CI): $0.85-0.90)$. The pooled specificity was 0.94 (95\% CI 0.93-0.96). The positive and negative likelihood ratios were 20.93 (95\% CI: 9.0648.34) and 0.15 (95\% CI: 0.10-0.22), respectively. SIS had good accuracy in the detection of all intrauterine abnormalities (area under the summary receiver operating curve $(\mathrm{sROC})=0.97 \pm 0.01)$. SIS also had a high pooled sensitivity and specificity in the detection of congenital uterine anomalies, 0.85 (95\% CI: 0.79-0.90) and 1.00 (95\% CI 0.99-1.00), respectively.SIS is a highly sensitive investigative modality and comparable to the gold standard tool, hysteroscopy in the detection of intrauterine abnormalities in subfertile women. SIS is a highly sensitive and specific test in the diagnosis of uterine polyps, submucous myomas, uterine anomalies and intrauterine adhesions and can be used as a screening tool for subfertile patients prior to IVF treatment. ${ }^{22}$

Bartkowiak R et al (2006) "The evaluation of uterine cavity with saline infusion sonohysterography and hysteroscopy in infertile patients". 68 infertile women, aged 27-42 were enrolled in the study. TVS, SIS and diagnostic HS were consecutively performed in every patient. The results of each method were compared. Sensitivity, specificity, positive predictive value and negative predictive values for TVS and SIS were obtained.Intrauterine pathologies were diagnosed in $25 \%$ of patients. TVS detected $6(37.5 \%)$ and SIS revealed 11 $(87.5 \%)$ of 13 intrauterine pathologies finally visualized at diagnostic hysteroscopy. TVS failed to visualize three submucous myomas, one endometrial polyp and two cases of septate uteri. All three cases of intrauterine synechiae were not detected with this method One submucous myoma and one endometrial polyp were not identified with SIS. The study group was, however, too limited to show statistically significant differences in diagnostic accuracy among TVS, SIS and HS.Saline infusion sonohysterography is simple, sensitive and inexpensive diagnostic method. The procedure is not time-consuming, causes minimal discomfort to the patient and may be performed without anesthesia in office settings. The method may be recommended for the diagnosis of intrauterine pathologies in infertile women. ${ }^{23}$

G. Obajimi et al (2016) conductes a study on "Intra-uterine lesions play an important role in the Routine saline infusion sonohysterography prior to assisted conception: a review of our initial experience". A descriptive retrospective study of 760 patients who had saline infusion sonohysterography prior to assisted conception, between January 2008 and December, 2010. Forty-six percent of the patients had intra-uterine pathologies. Submucous fibroids accounted for almost half (48.57\%) of the pathologies, followed by adhesions $(28.57 \%)$ and endometrial polyps $(22.86 \%)$. Complications arising from the procedure were minor and occurred in 26 patients (3.42\%). Abdominal cramps, vaginal bleeding and vaginal discharge occurred in $14(53.85 \%), 8(30.77 \%)$ and 4 $(15.38 \%)$ respectively. The average duration of the procedure was 6 minutes with a range of 4-9 minutes. Saline infusion sonohysterography is a reliable, cost effective and safe diagnostic tool in the evaluation of the uterine cavity prior to assisted conception. ${ }^{24}$

Zafar, Humaira et al (2019) conducted a study on "Diagnostic accuracy of saline infusion sonohysterography for evaluation of uterine cavity abnormalities in patients with abnormal uterine bleeding". Sixty patients presented with abnormal uterine bleeding were included in the study. Transvaginal ultrasound, SIS and hysteroscopy performed for evaluation of the uterine cavity for any abnormality.SIS helped in detection of intacavitary abnormalities in 39 out of 60 patients indicating sensitivity of $88.64 \%$ and specificity of $100 \%$ as hysteroscopy confirm the findings in all the patients. The NPV is $76 \%$ and PPV of $100 \%$ taking hysteroscopy as gold standard. The diagnostic accuracy of saline infusion sonography is $91 \%$. The addition of SIS to TVS significantly improved the sensitivity and specificity for detecting intracavitary pathology. It can be used as an alternative procedure whenever hysteroscopy is not available. ${ }^{25}$

Illan Tur Kapsa et al (2006) conducted a study on "An evaluation of uterine abnormalities by saline infusion sonohysterography in 1,009 women with infertility or abnormal uterine bleeding". Among the women in the infertility group, $16.2 \%(\mathrm{n}=97)$ were found to have intracavitary abnormalities, including polyps $(13.0 \%)$, submucous fibroids (2.8\%), and adhesions (0.3\%). Significantly, more patients in the AUB group $(39.6 \%, \mathrm{n}=162)$ revealed intracavitary abnormalities, including polyps $(29.8 \%)$, submucous fibroids $(9.0 \%)$, and adhesions $(0.7 \%)$. In contrast, significantly more uterine anomalies were found in the infertility group $(20 \%, \mathrm{n}=120)$ compared with the AUB group $(9.5 \%, \mathrm{n}=39)$. Arcuate uterus was the most common finding $(15 \% \mathrm{vs} .6 .4 \%$ of patients, respectively).An SIS procedure for infertility work-up revealed a substantial percentage of infertile patients with intracavitary abnormalities and uterine anomalies. Because the technique is safe, well tolerated, and feasible in an outpatient setting, SIS should be considered routinely in the early stage of infertility and AUB investigation. ${ }^{26}$

Van Dongen et al(2011) conducted a study on "Diagnostic hysteroscopy and saline infusion sonography in the diagnosis of intrauterine abnormalities: an assessment of patient preference". 
This study was conducted to assess whether women would prefer to undergo saline infusion sonography (SIS) or office hysteroscopy for the investigation of the uterine cavity. In a randomised controlled trial, 100 patients underwent SIS or office hysteroscopy for assessing patients' pain scores. After the investigation, 92 of them were asked to fill out an anonymous questionnaire addressing their preference regarding the method of evaluation and treatment of the uterine cavity. The questionnaire was completed by 113 women $(83.7 \%)$. Twenty-four $(21.2 \%)$ women would opt for SIS, whereas $52(46.0 \%)$ would opt for office hysteroscopy, and $37(32.7 \%)$ had no preference. If therapy would be necessary, $48.7 \%$ of the women would opt for an outpatient treatment, whereas $33.0 \%$ of the women would prefer treatment under general anaesthesia. Despite the fact that SIS is less painful, the majority of the women prefer office hysteroscopy. Additionally, therapy in an outpatient setting is preferred to a day case setting. ${ }^{27}$

Gunes M et al (2008) conducted a study on "Comparison of saline infusion sonography and histological findings in the evaluation of uterine cavity pathologies". Eighty-three women suspected of having endometrial cavity abnormalities were evaluated using saline infusion sonography. The results of this technique were compared with the histological evaluation reports of these women either with hysteroscopy or laparotomy prospectively. The positive predictive value (PPV) for endometrial polyps was 25\%; the negative predictive value (NPV) was $93 \%$; the sensitivity for endometrial polyps was $80 \%$ and the specificity was $87 \%$. The PPV, the NPV, the sensitivity and the specificity for submucous fibroids were $65,85,81$ and $89 \%$, respectively. Saline infusion sonography was a reliable and accurate method for investigations of the endometrium and uterine cavity with good correlation, with histological results of more invasive procedures. It can be a good alternative technique for the evaluation of uterine cavity abnormalities where office hysteroscopy is not available. ${ }^{28}$

Dijkhuizen FP et al (2000) conducted a study on "Comparison of transvaginal ultrasonography and saline infusion sonography for the detection of intracavitary abnormalities in premenopausal women". The results of 50 patients with abnormal uterine bleeding were evaluated. Histological examination revealed normal endometrial histology in 27 patients, submucus myomas in 13 patients and intracavitary polyps in 10 patients. The sensitivity of TVS in directly visualizing intracavitary abnormalities was $61 \%$ for a specificity of $96 \%$. The likelihood ratio of presence of an intracavitary abnormality was 16 and the likelihood ratio of absence of such a finding was 0.41 . When defining abnormality at TVS as direct visualization of an intracavitary abnormality or an increased endometrial thickness (cut - off level $5 \mathrm{~mm}$ ), the sensitivity of TVS was $87 \%$ for a specificity of $56 \%$, with corresponding likelihood ratios of 2 and 0.23 , respectively. The sensitivity and specificity of SIS was $100 \%$ and $85 \%$ with likelihood ratios of 6 and 0.0 , respectively. No intracavitary abnormality was missed by SIS.The diagnostic accuracy of SIS is higher than the accuracy of TVS. A combined approach using endometrial thickness measurement by TVS and, reserving SIS for patients with increased $(>5 \mathrm{~mm})$ endometrial thickness, or endometrium inadequately visualized on TVS, is the optimal method of reducing the hysteroscopy rate. ${ }^{29}$

Aydın, Önder, et al (2015) conducted a study on "The Role of Saline Infusion Sonohysterography in the Evalution of the Uterus of Infertile Patients." One hundred and two infertile patients who were referred to Süleymaniye Obstetrics and Gynecology Hospital because of infertility underwent saline infusion sonohysterography (SIS) at our clinic. The cases were informed about the procedure, and consent forms were obtained. Data was collected prospectively. Results were compared with a large patient cohort that was evaluated with only hysterography (HSG) and TvUSG for uterus assessment. Abnormal intracavitary findings were observed in $20.5 \%$ of the 102 infertile patients who had undergone SIS. This rate was $7.4 \%$ in the control group. Total abnormal intracavitary finding rate of the SIS group was significantly higher $(\mathrm{p}<0.01)$. The rates of the uterine anomalies were similar in two groups; $4.9 \%$ in the SIS group and $5.03 \%$ in the control group. There was no statistically significant difference between the uterine anomaly rates of the groups ( $p>0.05$ ). SIS is a practical, lowcost method that does not require specific equipment; in addition to these advantages, it is effective in the detection of intrauterine pathologies and for making differential diagnosis. In consideration of the higher frequency of uterine pathologies in infertile patients, SIS can be used as a routine test in infertility research

\subsubsection{Results}

In this systematic review we reviewed 15 article that was published between 2000 to2020. Extracted data from these reviewed article showed Two thousand infertile patients examined by saline infusion Sonography. From these 2000 patients 1400 women diagnose with abmormalities. A significant number of women with unexplained infertility present with unsuspected uterine abnormalities; therefore an assessment of the uterine cavity should be performed in all cases. Transvaginal ultrasound, SIS and hysteroscopy performed for evaluation of the uterine cavity for any abnormality. The addition of SIS to TVS significantly improved the sensitivity and specificity for detecting intra cavitary pathology. The sensitivity of saline infusion sonohysterography for detecting patency of the tubes was $100 \%$ and the specificity was $91 \%$. Saline infusion sono-hysterography was found to be a more reliable method of diagnosing tubal or uterine pathologies compared to hystero-salpingography for cases of infertility. .As an easy to perform, safe and well-tolerated procedure yielding high diagnostic accuracy, saline infusion SIS via this catheter seems to be superior to TVS and very close to HS. It may be used as the primary method for the detection of uterine abnormalities among postmenopausal women with abnormal uterine bleeding. 
It can be used as an alternative procedure whenever hysteroscopy is not available.

\section{Conclusion}

Saline infusion sono-hysterography was found to be a more reliable method of diagnosing tubal or uterine pathologies in infertile patients. SIS is a highly sensitive investigative modality and comparable to the gold standard tool, hysteroscopy in the detection of intrauterine abnormalities in infertile women. s. SIS plays an integral role in the baseline assessment of infertile women. SIS is relatively a short examination, less invasive, well tolerated by patients and more cost effective compared to hysteroscopy. SIS can be complementary to other imaging modalities in infertile women.

\section{References}

1. World Health Organization. Sexual and Reproductive Health. [Last accessed on 2018 Jul 23]. Available from: http://www.who.int/reproductivehealth/topics/infertility/perspective/en/index.html .

2. Patil M. Assessing tubal damage. J Hum Reprod Sci. 2009;2:2-11. [PMC free article] [PubMed] [Google Scholar]

3. National Institute for Health and Clinical Excellence: Fertility: Assessment and treatment for People with Fertility Problems. Nice Clinical Guideline 156. 2013. [Last accessed on 2018 Jul 23]. Available from: http://www.guidance.nice.org.uk/cg156 .

4. Seshadri, S., et al. "The evolving role of saline infusion sonography (SIS) in infertility." European Journal of Obstetrics \& Gynecology and Reproductive Biology 185 (2015): 66-73.

5. van Dongen H, Timmermans A, Jacobi CE, Elskamp T, de Kroon CD, Jansen FW. Diagnostic hysteroscopy and saline infusion sonography in the diagnosis of intrauterine abnormalities: an assessment of patient preference. Gynecol Surg 2011;8:65-70

6. Soares SR, Barbosa dos Reis MM, Camargos AF. Diagnostic accuracy of sonohysterography, transvaginal sonography, and hysterosalpingography in patients with uterine cavity diseases. Fertil Steril 2000;73:406-11.

7. Breitkopf D, Goldstein SR, Seeds JW. ACOG technology assessment in obstetrics and gynecology. Number 3, September 2003. Saline infusion sonohysterography. Obstet Gynecol 2003;102:659-62.

8. de Kroon CD, Jansen FW, Louwe LA, Dieben SW, van Houwelingen HC, Trimbos JB. Technology assessment of saline contrast hysterosonography. Am J Obstet Gynecol 2003;188:945-9.

9. AIUM practice guideline for ultrasonography in reproductive medicine. J Ultrasound Med 2009;28:128-37.

10. Spieldoch RL, Winter TC, Schouweiler C, Ansay S, Evans MD, Lindheim SR. Optimal catheter placement during sonohysterography: a randomized controlled trial comparing cervical to uterine placement. Obstet Gynecol 2008;111:15-21.

11. Zafaran F, Ahmadi F. Evaluation of intrauterine structural pathology by threedimensional sonohysterography using an extended imaging method. Int J Fertil Steril 2013;7:1-6

12. Moro F, Selvaggi L, Sagnella F, et al. Could antispasmodic drug reduce pain during hysterosalpingo-contrast sonography (HyCoSy) in infertile patients? A randomized double-blind clinical trial. Ultrasound Obstet Gynecol 2012;39: 260-5.

13. Jareethum R, Suksompong S, Petyim S, Prechapanich J, Laokirkkiat P, Choavaratana R. Efficacy of mefenamic acid and hyoscine for pain relief during saline infusion sonohysterography in infertile women: a double blind randomized controlled trial. Eur J Obstet Gynecol Reprod Biol 2011;155:193-8.

14. [14] Koskas M, Mergui JL, Yazbeck C, Uzan S, Nizard J. Office hysteroscopy for infertility: a series of 557 consecutive cases. Obstet Gynecol Int 2010;2010: 168096.

15. Sahu L, Tempe A, Gupta S. Hysteroscopic evaluation in infertile patients: a prospective study. Int J Reprod Contracept Obstet Gynecol 2012;1:37-41.

16. Izhar R, Mansuri FA, Armar NA, Tahir S. Diagnostic accuracy of Saline Infusion sonohystero-salpingography (SIS) as compared to Hystero-salpingography (HSG) in the assessment of sub-fertile women. J Pak Med Assoc. 2019 Jun;69(6):777-782. PMID: 31189281.

17. Singh V, Mishra B, Sinha S, Agrawal S, Thakur P. Role of Saline Infusion Sonohysterography in Infertility Evaluation. J Hum Reprod Sci. 2018;11(3):236-241. doi:10.4103/jhrs.JHRS_47_18

18. Izhar R, Husain S, Tahir S, Husain S. Incidence of intrauterine abnormalities in Pakistani women with unexplained infertility diagnosed via saline infusion sonography. J Ultrason. 2018;18(74):186-192. doi:10.15557/JoU.2018.0028

19. Bingol, B., Gunenc, M.Z., Gedikbasi, A. et al. Comparison of diagnostic accuracy of saline infusion sonohysterography, transvaginal sonography and hysteroscopy in postmenopausal bleeding. Arch Gynecol Obstet 284, 111-117 (2011). https://doi.org/10.1007/s00404-010-1604-0

20. Mohamed Rezk, Mohamed Shawky, The safety and acceptability of saline infusion sonography versus hysterosalpingography for evaluation of tubal patency in infertile women,Middle East Fertility Society Journal,Volume 20, Issue 2,2015,Pages 108-113,ISSN 1110-5690, 
21. B. Bingol, Z. Gunenc, A. Gedikbasi, H. Guner, S. Tasdemir \& B. Tiras (2011) Comparison of diagnostic accuracy of saline infusion sonohysterography, transvaginal sonography and hysteroscopy, Journal of Obstetrics and Gynaecology, 31:1, 54-58, DOI: 10.3109/01443615.2010.532246

22. Seshadri S, El-Toukhy T, Douiri A, Jayaprakasan K, Khalaf Y. Diagnostic accuracy of saline infusion sonography in the evaluation of uterine cavity abnormalities prior to assisted reproductive techniques: a systematic review and meta-analyses. Hum Reprod Update. 2015 Mar-Apr;21(2):262-74. doi: 10.1093/humupd/dmu057. Epub 2014 Dec 10. PMID: 25505226.

23. Bartkowiak R, Kaminski P, Wielgos M, Bobrowska K. The evaluation of uterine cavity with saline infusion sonohysterography and hysteroscopy in infertile patients. Neuro Endocrinol Lett. 2006 Aug;27(4):523-8. PMID: 16891990.

24. G Obajimil and b. Ogunkinle Routine saline infusion sonohysterography prior to assisted conception: a review of our initial experience, Ann Ibd. Pg. Med 2016. Vol.14, No.2 99-102

25. Zafar, Humaira, Mubashra Naz, and Umber Fatima. "Diagnostic accuracy of saline infusion sonohysterography for evaluation of uterine cavity abnormalities in patients with abnormal uterine bleeding." The Professional Medical Journal 26.06 (2019): 892-895.

26. Ilan Tur-Kaspa, Michael Gal, Michael Hartman, Jason Hartman, Alex Hartman, An evaluation of uterine abnormalities by saline infusion sonohysterography in 1,009 women with infertility or abnormal uterine bleeding,Fertility and Sterility,Volume 86, Issue 6,2006,Pages 1731-1735,ISSN 0015-0282,

27. van Dongen, H., Timmermans, A., Jacobi, C.E. et al. Diagnostic hysteroscopy and saline infusion sonography in the diagnosis of intrauterine abnormalities: an assessment of patient preference. Gynecol Surg 8, 65-70 (2011). https://doi.org/10.1007/s10397-010-0649-1

28. Gunes, M., Erol, O., Kayikcioglu, F. et al. Comparison of saline infusion sonography and histological findings in the evaluation of uterine cavity pathologies. Arch Gynecol Obstet 278, 513-516 (2008). https://doi.org/10.1007/s00404-008-0629-0

29. Dijkhuizen FP, De Vries LD, Mol BW, Brolmann HA, Peters HM, Moret E, Heintz AP (2000) Comparison of transvaginal ultrasonography and saline infusion sonography for the detection of intracavitary abnormalities in premenopausal women. Ultrasound Obstet Gynecol 15:372-376

30. Aydın, Önder, et al. "The Role of Saline Infusion Sonohysterography in the Evalution of the Uterus of Infertile Patients." (2015) ; 5: 102-6 